\title{
ESTUDO SOBRE A UTILIZAÇÃO DE RHODNIUS NEGLECTUS PARA XENODIAGNÓSTICOS REALIZADOS EM MARSUPIAIS (DIDELPHIS) *
}

\author{
Oswaldo Paulo Forattini ** \\ Maria das Dores Cotrim** \\ Eunice Aparecida Bianchi Galati ** \\ Silvia Branco Sarzana** \\ Cristina Ferez Cruz** \\ Nadja Helena Sabbag Van Dinteren** \\ Sabina Léa Davidson Gotlieb**
}

\begin{abstract}
RSPU-B/328
Forattin., O. P. et al. - Estudo sobre a utilização de Rhodnius neglectus para xenodiagnósticos reatizados em marsupiais (Didelphis). Rev. Saúde públ., S. Paulo, 10:335-45, 1976.

Resumo: Em marsupiais (Didelphis), portadores de infecção natural pelo T. cruzi, procurou-se levar a efeito xenodiagnósticos com o emprego de ninfas de Rhodnius neglectus $e$ de Triatoma infestans e através do exame periódico de lotes formados por cinco insetos. Pôde-se observar o melhor rendimento por parte do exame do intestino posterior, mediante dissecçāo. O tempo ótimo foi situado no intervalo correspondente a 15 e 20 dias, e a eficiência de $\mathrm{R}$. neglectus foi superior. Esse resultado, aliado à facilidade de sua manutenção em laboratório, sugere a utilização rotineira desse triatomineo para fins de xenodiagnóstico.
\end{abstract}

Unitermos: Tripanossomiase americana. Xenodiagnóstico. Rhodnius neglectus. Triatoma infestans. Reservatórios, tripanossomíase americana. Didelphis. Trypanosoma cruzi.

\section{INTRODUCAO}

O método do xenodiagnóstico, desde conhecer a melhor ocasião para fazê-lo, sua descrição por Brumpt $^{2}$ (1914), tem após a realização do repasto sangüíneo sido largamente utilizado com a finalida- infectante. No entanto, o estabelecimento de de detectar a presença de tripanososmíase americana, tanto em homens como em animais. Desde que a técnica implica o exame do conteúdo intestinal dos triatomíneos empregados, torna-se necessário desse período tem sofrido variaçóes consideráveis de acordo com diversos autores. Certamente, para isso, contribuem fatores múltiplos de variadas origens mas que, a grosso modo, podem ser atribuídos tan-

* Trabalho realizado pelo Departamento de Epidemiologia da Faculdade de Saúde Pública da USP, levado a efeito com o auxilio do Conselho Nacional de Desenvolvimento CientIfico e Tecnológico (Proc. CNPq n.o 10807).

* Do Departamento de Epidemiologia da Faculdade de Saúde Pública da USP - Avenida Dr. Arnaldo, 715 - São Paulo, SP - Brasil. 
FORATTINI, O. P. et al. - Estudo sobre a utilizaçāo de Rhodnius neglectus para xenodiagnósticos realizados em marsupiais (Didelphis). Rev. Saúde públ., S. Paulo, 10: $335-43,1976$.

to à cepa do Trypanosoma cruzi, como ao organismo do vertebrado submetido à prova e, também, ao triatomíneo utilizado.

O estudo de tempo ótimo foi, inicialmente, feito por Freitas ${ }^{4,5}$ (1947, 1950) que concluiu situando-o ao redor dos 60 dias. De certa forma, esse resultado veio confirmar orientação adotada anteriormente e que estabelecia esse período nos dias incluídos entre o $400^{\circ}$ e o $600^{\circ}$ (Dias ${ }^{3}$, 1940). Embora esse prazo fosse geralmente adotado, não existe ainda aspecto uniforme a respeito, e grande parte dos autores estabelecem, pelo menos, o primeiro exame, aos 30 dias ou mesmo anteriormente (Zeledón e Vieto ${ }^{13}$, 1957; Schenone e col. ${ }^{11}, 1968$; Marsden e col. ${ }^{7}$, 1969; Almeida e col. ${ }^{1}$, 1973; Schenone e col. ${ }^{12}$, 1974; Miles e col. ${ }^{8}, 1975$ ).

Em época mais recente, alguns estudos foram feitos com a finalidade de determinar o periodo de maior positividade no intestino dos insetos. Nesse sentido, Rohwedder e col. ${ }^{10}$ (1970), realizando exames periódicos dos triatomíneos utilizados, concluíram pela sua localização entre os 15 a 25 dias do repasto para Triatoma infestans. Resultado semelhante foi obtido por Patterson e Miles ${ }^{9}$ (1973) estabelecendo como ótimo o $25^{\circ}$ dia, em Rhodnius prolixus.

No decurso das investigações sobre os aspectos ecológicos da tripanossomíase americana, levadas a efeito na região $\mathrm{Su}-$ deste do Brasil, tivemos a contingência de aplicar o xenodiagnóstico em animais silvestres e domésticos. Surgiu daí a oportunidade de tentar simplificar o método combinando, para tanto a utilização de um triatomíneo facilmente colonizável em laboratório como o Rhodnius neglectus, com - estabelecimento de prazo mais breve para a realização do exame microscópico. Com esse objetivo programou-se série de experiências cujos resultados apresentamos.

\section{MATERIAL E METODOS}

As observaçōes foram realizadas com a utilização de marsupiais (Didelphis) naturalmente infectados e de triatomíneos das espécies Rhodnius neglectus e Triatoma infestans, colonizados em laboratório. Os trabalhos dividiram-se em duas etapas.

Primeira etapa - Utilizaram-se sete didelfídeos, seis Didelphis azarae e um D. marsupialis, capturados em várias localidades do Estado de São Paulo, Brasil (Tabela 1), e cuja positividade à infecção tinha sido previamente estabelecida mediante xenodiagnóstico preliminar.

Em ocasião simultânea, esses marsupiais foram oferecidos à alimentação de ninfas de $30^{\circ}$ e $4 .^{\circ}$ estádios de $R$. negelctus, em número de 150 insetos para cada animal. Decorrido o prazo de uma hora, separaram-se os triatomíneos que se apresentavam ingurgitados, desprezando-se os demais. A partir dessa data, os insetos foram examinados em intervalo regular de 84 horas, correspondente a cerca de 3,5 dias, escolhido arbitrariamente, até o total de 11 exames, ou seja, 38,5 dias decorridos do repasto sangüíneo.

Para o exame dos triatomíneos retirava-se, do conjunto correspondente a cada gambá, dez exemplares. Desse número, cinco foram examinados mediante a coleta de material fecal por simples compressão abdominal e os outros cinco através a dissecção e retirada do intestino posterior.

Segunda etapa - Face aos resultados obtidos na etapa anterior, procedeu-se a realização desta empregando-se ainda sete gambás. Desses animais, quatro foram os mesmos utilizados na primeira etapa, aos quais se acrescentaram três outros. Com exceção do exemplar de Didelphis marsupialis, que participou do primeiro grupo, todos os demais foram $D$. azarae (Tabela 1). A exemplo do que se fez anteriormente, a positividade dos novos animais 
FORATTINI, O. P. et al. - Estudo sobre a utiliząão de Rhodnius neglectus para xenodiagnósticos realizados em marsupiais (Didelphis). Rev. Saúde públ., S. Paulo, 10: $335-43,1976$.

TABELA 1

Procedência dos marsupiais Didelphis utilizados nas duas etapas

\begin{tabular}{|c|c|c|}
\hline $\mathrm{EXR} *$ & Espécie & Procedência (Estado de são Paulo - Brasil) \\
\hline $461 / 5$ & D. azarae & $\begin{array}{l}\text { Bairro Santo Antonio, municipio de São João da Boa Vísta, } \\
\text { captura peridomiciliar em VI. } 1975 \text {. }\end{array}$ \\
\hline $466 / 6$ & D. azarae & $\begin{array}{l}\text { Bairro do Pico, municipio de Taquarituba, captura silvestre } \\
\text { em VIII. 1975. }\end{array}$ \\
\hline $466 / 7$ & D. azarae & $\begin{array}{l}\text { Bairro do Pico, municipio de Taquarituba, captura sllvestre } \\
\text { em VIII. 1975. }\end{array}$ \\
\hline $466 / 8$ & D. azarae & $\begin{array}{l}\text { Bairro do Pico, municipio de Taquarituba, captura silvestre } \\
\text { em VIII. 1975. }\end{array}$ \\
\hline $466 / 10$ & D. azarae & $\begin{array}{l}\text { Bairro do Pico, municipio de Taquarituba, captura silvestre } \\
\text { em VIII. 1975. }\end{array}$ \\
\hline $466 / 11$ & D. azarae & $\begin{array}{l}\text { Bairro do Pico, municíplo de Taquarituba, captura silvestre } \\
\text { em VIII. 1975. }\end{array}$ \\
\hline $466 / 12$ & D. marsupialis & $\begin{array}{l}\text { Bairro do Pico, municipio de Taquarituba, captura silvestre } \\
\text { em VIII. 1975. }\end{array}$ \\
\hline $469 / 1$ & D. azarae & $\begin{array}{l}\text { Fazenda Baú, município de Santo Antonio da Alegria, cap- } \\
\text { tura silvestre em IX. } 1975 \text {. }\end{array}$ \\
\hline $469 / 5$ & D. azarae & $\begin{array}{l}\text { Fazenda Baú, município de Santo Antonio da Alegria, cap- } \\
\text { tura silvestre em IX. } 1975 \text {. }\end{array}$ \\
\hline $471 / 1$ & D. azarae & $\begin{array}{l}\text { Fazenda Baú, município de Santo Antonio da Alegria, cap- } \\
\text { tura peridomiciliar em IX. } 1975 \text {. }\end{array}$ \\
\hline
\end{tabular}

* Sigla para o número de registro no laboratório do Departamento de Epidemiologia da Faculdade de Saúde Pública da USP.

foi estabelecida através de xenodiagnóstico preliminar.

Seguindo a mesma orientação, esses marsupiais foram, simultaneamente, submetidos à alimentação de ninfas de $3 .^{\circ}$ e $4 .^{\circ}$ estádios de $R$. neglectus e de $T$. infestans, em lotes de 150 espécimens de cada triatomíneo para cada animal. Após uma hora de alimentação, foram separados os exemplares que se apresentavam repletos e desprezados os outros. A contar dessa data, os exames foram realizados com o mesmo intervalo regular de 84 horas, ou seja, cerca de 3,5 dias até completar o mesmo período de 38,5 dias contados a partir da alimentação. Para cada exame retirava-se um lote de dez ninfas do conjunto correspondente a cada gambá, constituído por cinco representantes de cada triatomíneo. Com base nos resultados obtidos na primeira etapa, procedeuse ao exame de todos esses insetos, mediante a dissecção e retirada do intestino posterior.

Os triatomíneos utilizados em ambas as fases supradescritas foram retirados de colônias mantidas em insetário e com as seguintes origens:

Rhodnius neglectus (RCT-20) - De uma fêmea coletada em cópula em palmeira macaúba (Acrocomia) no municipio de Pitangueiras, Estado de São Paulo, Brasil (III. 1969).

Triatoma infestans (RCT-2) - De vários adultos procedentes de coletas domiciliares em diversos municípios do Estado 
FORATTINI, O. P. et al. - Estudo sobre a utilização de Rhodnius neglectus para xenodiagnósticos realizados em marsupiais (Didelphis). Rev. Saúde públ., $\mathbf{S}$. Paulo, 10: $335-43,1976$.

de São Paulo. Brasil (II. 1968 a II. $1973)$.

Em todos os exames considerou-se como positivo aquele no qual a microscopia revelou a presença de formas vegetativas de protozoário em movimentação ativa.

\section{RESULTADOS}

De acordo com o exposto. caberá apreciar os resultados obtidos em cada um dos estágios. No primeiro, com o objetivo de observar possível associação entre o tempo e o processo de exame. No segundo, procurando confirmar esse aspecto comparando as duas espécies triatomínicas.

Primeira etapa - As Tabelas 2 e 3 fornecem idéia dos resultados conseguidos, respectivamente, com a compressão abdominal e a dissecção do intestino posterior das ninfas de $R$. neglectus. Pode-se ver a ocorrência de maior número de insetos positivos a partir do 6." exame, corres- pondente a 21 dias do repasto, para as fezes obtidas por compressão do abdome. Quanto ao exame direto do conteúdo do intestino posterior, obtido por dissecção do inseto, verifica-se maior número de positivos desde, praticamente, o $20^{\circ}$ exame, ou seja, o sétimo dia contado da hematofagia. Testando-se a proporção desse tipo de resultados obtidos pelo uso das duas técnicas, observou-se maior positividade para a dissecção, estatisticamente significante, a um nível de $5 \%$ quando do $5 .^{\circ}$ exame $(K=5,62)$. No entanto, não houve diferença estatística $(K=1,01)$ quando do $6 .^{\circ}$ exame, ao mesmo nível de $5 \%$. sendo, nessa oportunidade, praticamente equivalente o rendimento dos dois processos.

Verifica-se também a ocorrência de variaçōes individuais, sendo a mais marcante a referente ao gambá 466/12, o único representante aqui da espécie Didelphis marsupialis, e cuja positividade parece ter sido bem mais demorada do que os demais. para ambas as técnicas.

TA B E A 2

Resultados dos xenodiagnósticos realizados com Rhodnius neglectus em Didelphis naturalmente infectados Exames periódicos mediante coleta do material por compressão abdominal

\begin{tabular}{|c|c|c|c|c|c|c|c|c|c|c|c|}
\hline \multirow{2}{*}{ EXR* } & \multicolumn{11}{|c|}{ Exames periódicos (dias) ** } \\
\hline & $\begin{array}{c}1.0 \\
(3,5)\end{array}$ & $\begin{array}{c}2.0 \\
(7,0)\end{array}$ & $\begin{array}{c}3.0 \\
(10,5)\end{array}$ & $\begin{array}{c}4.0 \\
(14,0)\end{array}$ & $\begin{array}{c}5 .^{\circ} \\
(17,5)\end{array}$ & $\begin{array}{c}6.0 \\
(21,0)\end{array}$ & $\begin{array}{c}70^{\circ} \\
(24,5)\end{array}$ & $\begin{array}{c}80^{\circ} \\
(28,0)\end{array}$ & $\begin{array}{c}9.0 \\
(31,5)\end{array}$ & $\begin{array}{c}10.0 \\
(35,0)\end{array}$ & $\begin{array}{c}110^{\circ} \\
(38,5)\end{array}$ \\
\hline $461 / 5$ & - & 3 & 1 & 1 & 1 & 5 & 3 & 5 & 5 & 5 & 5 \\
\hline $466 / 6$ & 一 & 3 & 1 & 3 & 3 & 5 & 3 & 4 & 5 & 5 & 5 \\
\hline $466 / 7$ & - & - & 1 & 1 & 2 & 5 & 3 & 5 & 5 & 5 & 5 \\
\hline $466 / 8$ & - & 1 & 1 & 2 & 3 & 5 & 3 & 5 & 5 & 5 & 5 \\
\hline $466 / 10$ & - & 1 & - & 1 & $I$ & 5 & 3 & 5 & 4 & 5 & 3 \\
\hline $466 / 11$ & 2 & 3 & 2 & 3 & 1 & 5 & 3 & 5 & 4 & 5 & 4 \\
\hline $466 / 12$ & - & 一 & 一 & - & 1 & 4 & 3 & 5 & 5 & 5 & 4 \\
\hline Total & 2 & 11 & 6 & 11 & 12 & 34 & 21 & 34 & 33 & 35 & 31 \\
\hline
\end{tabular}

* Vide Tabela 1.

* Número de insetos positivos em cada lote de 5 exemplares. 
FORATtini, O $P$. et al. - Estudo sobre a utilizacão de Rhodnius neglectus para xeno. diagnósticos realizados em marsuplais (Didelphis). Rev. Saude pribl., S. Paulo, 10: 335-43. 1976.

$T A B E L A 3$

Resultados dos xenodiagnosticos realizados com Rhodnus neglectus em Didelphis naturalmente infectados. Exames periodicos mediante coleta do material por disseccão do intestino posterior $11^{\text {a }}$ etapa)

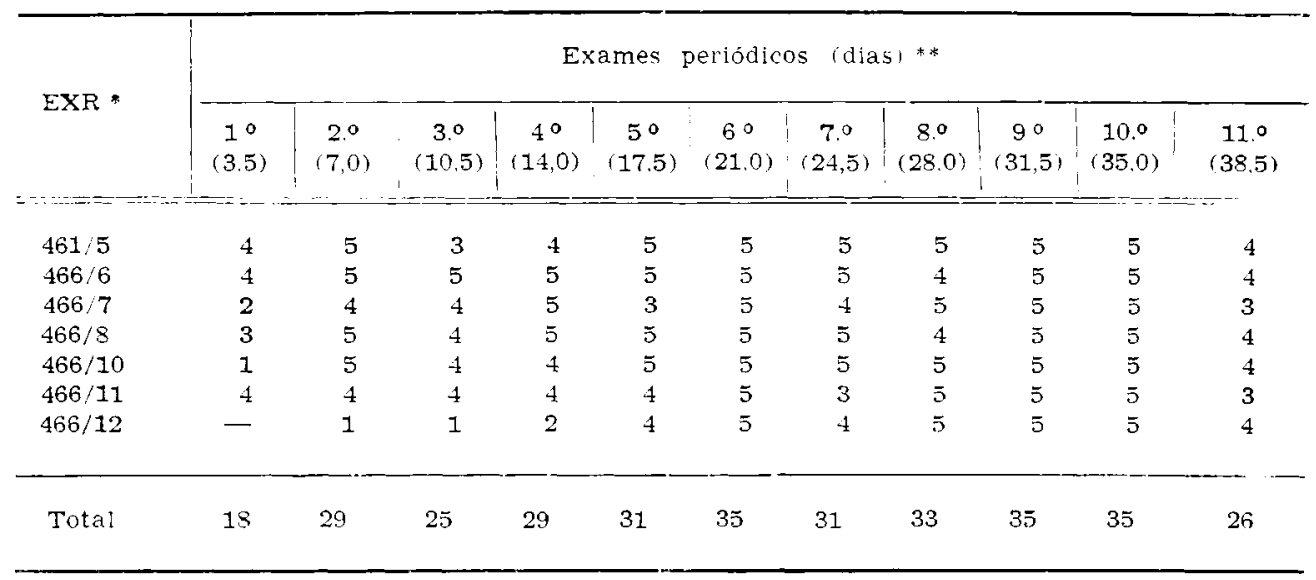

* Vide Tabela 1

* Número de insetos positros em cada lote de 5 exemplares

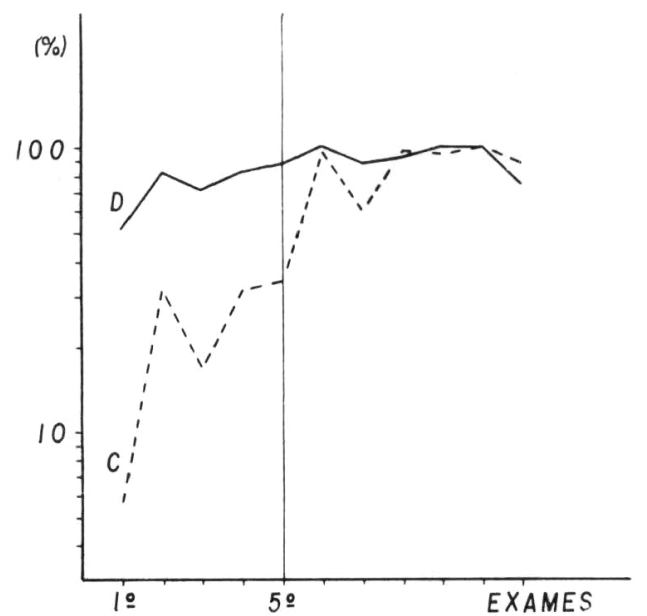

Fig. 1 - Percentual de ninfas de Rhodnius neglectus positivas (1.a etapa), sobre o total de cada exame, por compressão abdominal (C) e por disseção do intestino posterior (D). Nota-se a maior tendência à positividade a partir do $50^{\circ}$ exame (17.5 dias) e o predomínio geral do segundo método sobre o primeiro.
Calculando-se o percentual de insetos que se revelaram como positivos no total de cada exame. obteve-se o aspecto do: gráficos da Figura 1. Pode-se ali observar a maior rapidez do processo haseado na dissecção do intestino posterior e a maior positividade até o $50^{\circ}$ exame correspente ao periodo de 17.5 dias.

Segunda etapa - Pela análize dos dados constantes das Tahelas 4 e 5 . pode-se observar. em linhas gerais. a repetição do comportamento de $R$. neglectus. com a precocidade dos resultados positivos desde. praticamente. o 3." exame. Ao nível do 5." exame dá-se nova ascensão desse rendimento.

Comparando-se os dados obtidos com esse triatomíneo e Triatoma infestans, observou-se maior proporção de positivos para Rhodnius neglectus. As diferenças foram estatisticamente significantes a um nivel de $5 \%$. quer seja para o $5 .^{\circ}$ exame $\mathrm{K}=1.751$. quer seja para o $6 . " \mathrm{~K}=$ 
FORATTINI, O. P. et al. - Estudo sobre a utilização de Rhodnius neglectus para xenodiagnósticos realizados em marsupiais (Didelphis). Rev. Saúde públ., S. Paulo, 10: $335-43,1976$.

TABELA 4

Resultados dos xenodiagnósticos realizados com Rhodnins neglectus em Didelphis naturalmente infectados. Exames periódicos mediante coleta do material por dissecção do intestino posterior ( $2^{\text {a }}$ etapa)

\begin{tabular}{|c|c|c|c|c|c|c|c|c|c|c|c|}
\hline \multirow{2}{*}{$\mathrm{EXP} *$} & \multicolumn{11}{|c|}{ Exames periódicos (dias) ** } \\
\hline & $\begin{array}{c}1.0 \\
(3.5)\end{array}$ & $\begin{array}{c}2.0^{\circ} \\
(7,0)\end{array}$ & $\begin{array}{c}3^{\circ} \\
(10,5)\end{array}$ & $\begin{array}{c}40^{\circ} \\
(14,0)\end{array}$ & $\begin{array}{c}50^{\circ} \\
(17,5)\end{array}$ & $\begin{array}{c}6 .^{\circ} \\
(21,0)\end{array}$ & $\begin{array}{c}7.0 \\
(24,5)\end{array}$ & $\begin{array}{c}80^{\circ} \\
(28,0)\end{array}$ & $\begin{array}{c}9.0 \\
(31,5)\end{array}$ & $\begin{array}{c}10.0 \\
(35,0)\end{array}$ & $\begin{array}{c}11.0^{\circ} \\
(38,5)\end{array}$ \\
\hline $461 / 5$ & 4 & 5 & 5 & 4 & 4 & 4 & 5 & 5 & 5 & 5 & 4 \\
\hline $466 / 7$ & 一 & 3 & 4 & 4 & 4 & 5 & 5 & 4 & 4 & 4 & 4 \\
\hline $466 / 11$ & 3 & 4 & 5 & 5 & 5 & 5 & 5 & 5 & 5 & 2 & 4 \\
\hline $466 / 12$ & 一 & 1 & 5 & 3 & 3 & 5 & 5 & 4 & 4 & 3 & 3 \\
\hline $469 / 1$ & 5 & 3 & 5 & 5 & 5 & 5 & 3 & 5 & 5 & 5 & 5 \\
\hline $469 / 5$ & 5 & 2 & 5 & 5 & 5 & 5 & 5 & 5 & 5 & 5 & 4 \\
\hline $471 / 1$ & 5 & 4 & 5 & 5 & 4 & 5 & 3 & 5 & 1 & 4 & 4 \\
\hline Total & 22 & 22 & 34 & 31 & 30 & 34 & 31 & 33 & 29 & 28 & 28 \\
\hline
\end{tabular}

* Vide Tabela 1.

* Número de insetos positivos em cada lote de 5 exemplares.

TABELA 5

Resultados dos xenodiagnósticos realizados com Triatoma infestans em $D$ idelphis naturalmente infectados. Exames periódicos mediante coleta do material por disseção do intestino posterior

\begin{tabular}{|c|c|c|c|c|c|c|c|c|c|c|c|}
\hline \multirow{2}{*}{$\mathrm{EXR}$ * } & \multicolumn{11}{|c|}{ Exames periódicos (dias) ** } \\
\hline & $\begin{array}{c}1.0 \\
(3,5)\end{array}$ & $\begin{array}{c}2 .{ }^{\circ} \\
(7,0)\end{array}$ & $\begin{array}{c}30^{\circ} \\
(10,5)\end{array}$ & $\begin{array}{c}4 .^{\circ} \\
(14,0)\end{array}$ & $\begin{array}{c}5 .^{\circ} \\
(17,5)\end{array}$ & $\begin{array}{c}6^{\circ} \\
(21,0)\end{array}$ & $\begin{array}{c}7^{\circ} \\
(24.5)\end{array}$ & $\begin{array}{c}8 .^{\circ} \\
(28,0)\end{array}$ & $\begin{array}{c}9.0 \\
(31,5)\end{array}$ & $\begin{array}{c}10.0 \\
(35,0)\end{array}$ & $\begin{array}{c}11.0 \\
(38,5)\end{array}$ \\
\hline $461 / 5$ & 3 & 3 & 4 & 3 & 4 & 4 & 5 & 5 & 5 & 4 & 4 \\
\hline $466 / 7$ & - & 一 & - & 3 & 2 & 2 & 3 & 4 & 1 & 1 & 2 \\
\hline $466 / 11$ & 3 & 2 & 4 & 5 & 4 & 5 & 5 & 4 & 4 & 3 & 3 \\
\hline $466 / 12$ & - & - & 2 & 2 & 1 & 3 & 3 & 4 & 5 & 5 & 3 \\
\hline $469 / 1$ & 3 & 5 & 2 & 4 & 4 & 2 & 4 & 3 & 3 & 3 & - \\
\hline $469 / 5$ & 4 & 4 & 4 & 4 & 4 & 4 & 5 & 5 & 4 & 3 & 3 \\
\hline $471 / 1$ & 4 & 5 & 4 & 4 & 5 & 5 & 4 & 5 & 5 & 4 & 4 \\
\hline Total & 17 & 19 & 20 & 25 & 24 & 25 & 29 & 30 & 27 & 23 & 19 \\
\hline
\end{tabular}

* Vide Tabela 1.

* Número de insetos positivos em cada lote de 5 exemplares.

2,191. De maneira geral, esse aspecto manteve-se ao longo das observações.

Calculando-se o percentual de ninfas que se revelaram positivas em relação ao total de cada exame, obteve-se o aspecto dos gráficos da Figura 2. Pode-se observar a maior rapidez e superior rendimento do $R$. neglectus em relação ao $T$. infes- 
FORATTINI, O. P. et al, - Estudo sobre a utilização de Rhodnius neglectus para xenodiagnóstlcos realizados em marsupiais (Didelphis). Rev. Saúde públ., S. Paulo, 10: $335-43,1976$.

tans, com a utilização do processo de dissecção do intestino posterior. Da mesma forma que na etapa anterior obteve-se resultado significante a partir do $50^{\circ}$ exame, ou seja, com cerca de 17,5 dias.

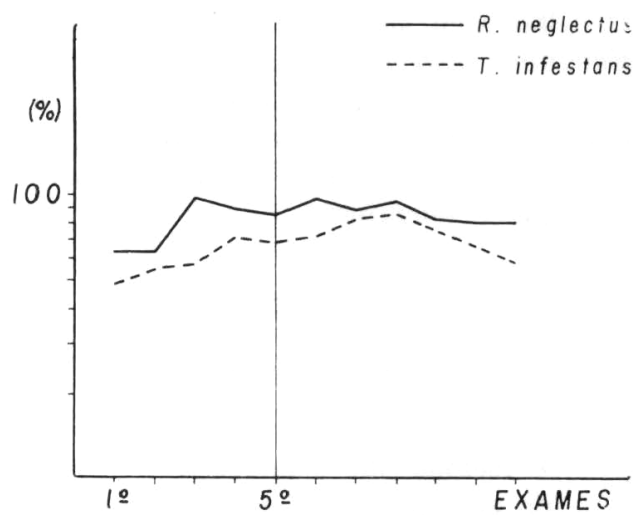

Fig. 2 - Percentual de ninfas de Rhodnius neglectus (2. etapa) e de Triatoma infestans positivas, por dissecçáo do intestino posterior. Nota-se geral predomínio das primeiras sobre as segundas, com tendência à equivalência após o $5 .^{\circ}$ exame $(17,5$ dias $)$.

\section{COMENTARIOS}

Como já foi mencionado anteriormente, um dos principais objetivos deste estudo residiu no estabelecimento de menor periodo para a positividade do xenodiagnóstico, que permitisse razoável segurança. Certamente, são numerosos os fatores que intervêm no resultado obtido com esse método, influindo direta ou indiretamente no desenvolvimento do $T$. cruzi no intestino do triatomíneo. Em vista disso, dificilmente será possível isolar um deles livrando-o da influência dos demais. No entanto, mesmo que de maneira aproximada, algo pode ser deduzido, como no caso dos dados conseguidos neste trabalho.

Inicialmente, as informações obtidas na execução da primeira etapa sugerem a superioridade do processo de dissecção do intestino posterior sobre o do simples exa- me de material fecal obtido por compressão abdominal do inseto. Essa vantagem manifestou-se principalmente pela precocidade. Assim, o 5. ${ }^{\circ}$ exame realizado aos 17,5 dias apresentou-se significantemente com maior rendimento. Este tornou-se equivalente para ambas as técnicas por ocasião do $6 .^{\circ}$ exame levado a efeito aos 21 dias. Tais aspectos sugerem a possibilidade de adotar a dissecção em época anterior a esse período. Poderá, pois, ser fixada no intervalo entre os 15 e 20 dias do repasto sangüíneo.

Quanto à comparação entre as cepas de $R$. neglectus e $T$. infestans, parece ter havido maior vantagem para aquele. tanto na positividade como no menor tempo. A partir do $5 .^{\circ}$ exame, os resultados para ambos os insetos tenderam a se aproximar. De qualquer maneira, se for considerado o mesmo período referido no parágrafo anterior, pode-se admitir a sua confirmação nesta segunda série de observações, a despeito de algumas discrepâncias. Estas ocorreram principalmente nos exames mais precoces como se pode observar nas Tabelas 3 e 4 e pelas curvas obtidas com $R$. neglectus (Figs. 1(D) e 2). Assim sendo, levando-se em conta a multiplicidade de fatores que podem influir em tais resultados, será oportuno considerar as condições em que foram levadas a efeito as experiências supradescritas.

Inicialmente, as cepas de $T$. cruzi foram todas de origem animal, especificamente de didelfídeos, e totalmente desconhecidas quanto às suas características. Não será possível dizer qual o número exato $e$, assim, sua influência foge completamente ao controle nos resultados obtidos. Da mesma forma, os gambás ( $D i$ delphis) utilizados, além de pertencerem a duas espécies, tiveram procedência variada. Assim, à somatória da ação de todos esses fatores deve ser atribuída, necessariamente, grande parte da variabilidade de dados. Já se chamou a atenção para, no caso dos vários marsupiais con- 
FORATTINI, O. P. et al. - Estudo sobre a utilização de Rhodnius neglectus para xenodiagnósticos realizados em marsupiais (Didelphis). Rev. Saúde públ., S. Paulo, 10: $335-43,1976$.

siderados individualmente, 0 que se observou com o n. ${ }^{\circ} 466 / 12$, único representante de $D$. marsupialis, que parece ter discrepado sensivelmente dos demais, todos pertencentes à espécie $D$. azarae. Vale a pena assinalar também que, no critério de positividade, adotou-se a presença de formas do parasito em mobilidade ativa. $\mathrm{Na}$ verdade, ocorreu, em várias oportunidades, principalmente após os $99^{\circ}$ e 10. exames, a constatação de formas imóveis, aparentemente mortas, embora o resultado fosse rotulado como negativo. Mas, de qualquer forma, compreende-se que tais aspectos deixassem de ser controlados. É que o objetivo principal residiu no diagnóstico mais rápido de infecçōes naturais e, por conseguinte, os marsupiais representaram o papel de portadores de infecçōes de variadas procedências.

Quanto aos triatomineos empregados, a focaliazção do Rhodnius neglectus deveu-se à facilidade com que esse inseto se mantém e à rapidez com que se reproduz em condições de laboratório. A comparação dos seus resultados com aqueles obtidos em Triatoma infestans sofre o prejuízo resultante da constituição das duas colônias. Com efeito, enquanto a primeira foi formada por um clone, a segunda reuniu indivíduos de várias origens. Daí a provável influência desse fator, possivelmente traduzida por variaçóes na sensibilidade à infecção, por parte dos representantes de $T$. infestans empregados. Com efeito, tem sido de longa data considerado que o $T$. cruzi tenderia a se desenvolver melhor em triatomíneos $\mathrm{da}$ mesma procedência (Little e col. ${ }^{6}, 1966$ ). Embora isso seja admitido a nível de espécie, não se pode afastar a possibilidade que o mesmo ocorra em relação a grupos infra-específicos. Contudo, em que pese a existência de tais variáveis, forçoso é levar em conta que o tempo ótimo de evolução do parasito no intestino posterior parece ser menor do que se supunha. Como foi citado, algumas observações têm indicado o tempo de cerca de 25 dias para Rhodnius prolixus e, mesmo para $T$. infestans, esse período não iria além dos 20 dias (Rohwedder e col. ${ }^{10}, 1970$; Patterson e Miles ${ }^{9}, 1973$ ). Dessa maneira, embora deva admitir-se a presença de numerosas variáveis, os resultados deste trabalho vêm possibilitar o emprego de Rhodnius neglectus para o xenodiagnóstico, pelo menos para animais silvestres, reduzindo o tempo de exame para o intervalo de 15 a 20 dias, contados a partir do repasto sangüíneo.

\section{CONCLUSOES}

Face ao exposto, podemos concluir o que segue.

1. O método do xenodiagnóstico pode ser aplicado para animais silvestres, pelo menos a Didelphis, com o emprego de ninfas de $3 .^{\circ}$ e $4 .^{\circ}$ estádio de Rhodnius neglectus.

2. O melhor rendimento poderá ser obtido mediante o exame do conteúdo intestinal posterior obtido após dissecção.

3. O tempo ótimo parece estar incluído no intervalo compreendido entre 15 e 20 dias após o repasto sangüíneo, mediante a utilização de, pelo menos, cinco insetos.

4. Além da redução do prazo para exame, a utilização de $R$. neglectus apresenta a vantagem de se tratar de triatomíneo facilmente colonizável e de elevada taxa de reprodução em condiçôes de laboratório.

5. De maneira geral, nesta série de observaçōes, o $R$. neglectus mostrou-se superior ao Triatoma infestans no que respeita seu desempenho para fins de xenodiagnóstico.

6. Tais resultados abrem possibilidade de sua aplicação em casos humanos da infecção chagásica. Contudo, isso somente poderá ser feito após a realização de novas observaçôes. 
FORATTINI, O. P. et al - Estudo sobre a utilizaçāo de Rhodnius neglectus para xenodiagnósticos realizados em marsupiais (Didelphis). Rev. Saúde púbt., S. Paulo, 10: $335-43,1976$.

Forattini, O. P. et al. - LThe utilization of Rhodnius neglectus in xenodiagnosis: a study performed on opossumsl. Rev. Saúde públ., S. Paulo, 10: 335-43, 1976.

SuMMARY: Third and fourth instars of Rhodnius neglectus and Triatoma infestans were fed on wild opossums (Didelphis) with natural infeccions by Trypanosoma cruzi and examined at regular 3.5 days intervals. Best results were obtained with rectum examinations and the infeccion was more easily detected from the 15 th to the 20th days. R. neglectus showed better performance than $\mathrm{T}$. infestans and so its utilization in the xenodiagnosis technique is suggested.

UNITERMs: American trypanosomiasis. Xenodiagnosis. Rhodnius neglectus. Triatoma infestans. Trypanosomiasis reservoirs. Didelphis. Trypanosoma cruzi.

\section{REFERENCIAS BIBLIOGRAFICAS}

1. AIMEIDA, S. P. et al - Verificação da susceptibilidade à infecção por Trypanosoma cruzi, dos estágios evolutivos de Rhodnius neglectus. Rev. bras. Biol., 33:43-52, 1973.

2. BRUMPT, E. - O xenodiagnóstico: aplicação ao diagnóstico de algumas infeccooes parasitárias e em particular à trypanosomose de Chagas. An. paut. Med. Cirurg., 3:97-102, 1914.

3. DIAS, E, - Técnica do xenodiagnóstico na moléstia de Chagas. Mem. Inst. Osw. Cruz, 35:335-42, 1940.

4. FREITAS, J. I. P. de - Observacōes sobre o tempo ótimo para exame de triatomídeos empregados em xenodiagnóstico. Folia clín. biot., S. Paulo, $16: 180-5,1950$.

5. FREITAS, J. L P. de - Contribuigño para o estudo do diagnóstico da moléstia de Chagas por processos de laboratório. São Paulo, 1947. [Tese - Faculdade de Medicina USP].

6. LITTLE, J. W. et al. - A study on the susceptibility of triatomid bugs to some mexican strains of Trypanosoma cruzi. J. Med. Entomol., 3:252-5, 1966 .

7. MARSDEN, P. D et al. - Some observations on xenodiagnosis with Rhodnius prolixus and Triatoma infestans in human infections with Bahian strains of Trypanosoma cruzi. Trans. roy. Soc, trop. Med. Hyg., 63:425-6. 1969 .
8. MILES, $M$ A et al. - A comparison of Rhodnius prolixus, Trlatoma infestans and Panstrongylus megistus in the xenodiagnosis of a chronic Trypanosoma (Schizotrypanum) cruzi infection in a rhesus monkey (Macaca. mullatta). Trans. roy. Sor. trop. Med. Hyg., 69:377-82, 1975 .

9. PATTERSON, J. W. \& MILES, M. A. Trypanosome density in the rectum of Rhodnius prolixus infected with T. cruzi. Trans, roy, Soc. trop. Med. Hyg., 67:442, 1973.

10. ROHWEDDER, R. W. et al. - Apartes al método del examen del xenodiagnóstico previo licuado de los triatomineos. Bol. chil. Parasit,, 25: 106-10, 1970 .

11. SCHENONE, H. et al, - Valor del xenodiagnóstico en la infección chagásica crónica. Bol. chil, Parasit., 23:149-54, 1968 .

12. SCHENONE, $\mathrm{H}$. et al. - Bases y rendimiento del xenodiagnóstico en la infección chagásica humana. Bol. chil. Parasit., 29:24-6, 1974.

13. ZELEDóN, R. \& VIETO, P. L. - Susceptibilidad de varias espécies de triatominos a una cepa costarricense de Schizotrypanum cruze Chagas, 1909 Rev. Biol. trop., S. José, 5: 195-9. 1957.

Recebido para publicasão em 02/06/1976 Aprovado para publicacino em 14/06/1976 